but the particles continually shifted about, as could be explained by the repeated death of dust-laden phagocytes with release of their burden, which was then incorporated by new cells.

After a single administration of dust, phagocyte counts in saline washed into and out of rat lungs demonstrated that the number of cells was related to the quantity of dust eliminated. The half-life of this phase of elimination by phagocytosis to the bronchial epithelium was about $17 \mathrm{hr}$. The proportion of dust eliminated in this way depended on the nature of the dust, but the time-constant did not; it did not appear to matter whether the dust was given by inhalation or by intratracheal injection.

Another series of experiments confirmed the destructive action of silica dusts on macrophages and once again led to the suggestion that the damage inflicted on the cells is the first step in the chain of causation of silicosis. The effect is believed not to be cytotoxic, but to comprise an activation either of a serum complement absorbed on the outside of the cells or of an enzyme, possibly a non-specific esterase, within the cells.

Examination of lung residues from 50 asbestosis cases was reported, with the conclusion that a fibrogenic agent was liberated when asbestos bodies break up. The deposition of an asbestos fibre in lung tissue is followed by its becoming enclosed in a protein sheath which passes through a sequence of changes and finally segments, after $10-15$ years, with fracture of the contained fibre. The fragments of the sheath are collected by interstitial macrophages and fibrosis is marked in regions where this occurs. The time sequence of clinical events and of lung content of fibres exceeding $26 \mu$ in length supports the argument.

Papers on dust-sampling included the description of an ingenious centrifugal device called the 'conicycle', which collects only particles between upper and lower. limits of falling speed. It has been engineered as a practical sampling tool for coal mines and is being tried out underground.

The relationship between, the acceptance curve of a sampling instrument designed to control dust conditions relevant to the incidence of pneumoconiosis, and the actual deposition-particle-size curve of the human respiratory tract was given more emphasis by the engineers than our crude and incomplete knowledge of the latter can possibly justify.

Arguments in favour of selecting finer particles than the 50 per cent at $5 \mu$ diameter and unit density, which at present forms the basis of British selective sampling practice, are therefore poorly based. The opposite view was also put forward, that all dust should be sampled, irrespective of particle size; besides being physically impossible this would encourage the collection of large quantities of coarse dust which has no conceivable bearing on pneumoconiosis because, when inhaled, it would not penetrate beyond the bronchi, and larger bronchioles.

The papers and discussions will be published by Pergamon Press in due course.

\section{N. Davies}

\title{
CARDIOVASCULAR EFFECTS OF NICOTINE AND OF SMOKING
}

$D^{2}$ URING March 23-25 a symposium on the Cardiovascular Effects of Nicotine and of Smoking was held by the Now York Academy of Sciences. The papers presented covered more than the cardiovascular effects and included other interesting observations. For example, nicotine is found not only in the tobaceo plant, but it also oceurs widely in the plant kingdom. It is produced in the root system of the tobacco plant, and the growth of the roots is very closely related to the rate of nicotine production, from which R. F. Dawson (Columbia) and his colleagues conclude that the same reaction step limits both processes. Nicotine arises from the precursors nicotinic acid and ornithine. From the root it is transported primarily to tho leaves, where it accumulates during the active life of the plant. It is believed to be a terminal product with no appreciable physiological activity for the plant. Such a conclusion seems to an animal physiologist to be highly unlikely.

Nicotine is absorbed not only by those who smoke but also by those who take snuff and those who chew tobacco. The figures given for the amount of nicotine absorbed when smoking one cigarette vary according to the degree to which smoke is inhaled. They range from estimates as low as $0.3 \mathrm{mgm}$. to others as high A.s $1.8 \mathrm{mgm}$. Since the intravenous injection of $0.7 \mathrm{mgm}$. nicotine (base) produces unpleasant symptoms, the higher figures must be regarded with doubt.

Nicotine taken in the form of snuff has been estimated to amount to $20-60 \mathrm{mgm}$. a day, and that absorbed as a result of chewing has been estimated as 10-90 mgm, a day (Larsen, Richmond, Virginia.). It is obvious that the taking of snuff and the chewing of tobacco offer much less risk of producing cancer of the lung.

The metabolism of nicotine is not yet fully known, and further work is badly noeded. Nicotine is found in the urine excreted unchanged, together with related substances (McKennis, Richmond, Virginia). Oxidation of the pyrrolidine ring appears to be a major step. The lactam (-)-cotinine which arises from the motabolism of $(-)$-nicotine is oxidized, and in the dog there is hydroxylation and methylation. It seems possible that the metabolism of nicotine changes with advancing years, some processes declining in rate or ceasing altogether. If the toleration of nicotine depends on a high rate of breakdown, the observation that some older people stop smoking who smoked much when young may depend on such a decline.

Nicotine has been known to cause poisoning in those using it as an insecticide. Travell (Cornell) has shown that its absorption through the skin is greatly affected by the $p \mathrm{H}$ of the solution. At $\mathrm{a} p \mathrm{H}$ of $5 \cdot 0$ or less it is very little absorbed, but at an alkaline $p \mathrm{H}$ it is readily absorbed. This holds true for the membrane of the stomach and of the bladder. Nicotine excreted in the urine may bo absorbed from the bladder into the blood if the urine is alkaline, and may produce toxic effects. The speed of absorption depends on the concentration of free base. On the cardiovascular system, nicotine has long been believed to owe its action to stimulation of the 
autonomic ganglia. Langley applied nicotine to ganglia in order to determine the effect of stimulating the cells present in the ganglia. Recently, attention has been directed to various peripheral effects of nicotine at sites where ganglia are not present. Thus, nicotine has a two-fold effect in the isolated heart. It causes first inhibition of contractions, and second acceleration and augmentation. The inhibition is excluded in the presence of atropine, but the acceleration is unaffected. In the heart there is a store of noradrenaline which can be extracted from the heart. Hitherto the existence of this store has been neglected by physiologists. It is now evident that the acceleration of the heart caused by nicotine is due to the release of some of the noradrenaline from the store. This follows from the observation that when there is no store of noradrenaline, nicotine loses its accelerating action (Burn and Rand, Oxford). Treatment of the animal with reserpine causes a disappearance of the store at the end of a few hours. The rise in heart-rate which follows smoking is thus probably explained by an action of nicotine on the heart itself releasing noradrenaline from storage sites in the heart.

In 1940 it was shown by Grace Roth and her colleagues that when a person smoked a cigarette in a warm room there was a large fall in skin temperature. This fall was believed to result from a constriction of the skin vessels caused by a stream of impulses passing down the vasoconstrictor nerves. However, observations have recently been made in the isolated ear of the rabbit, which contains no ganglia. These have shown that when the vessels of the ear are perfused with a modified Ringer's solution, the outflow being measured with a sensitive recorder, the injection of $3 \mu \mathrm{gm}$. of nicotine into the fluid about to enter the ear causes vasoconstriction. The outflow from the ear veins is greatly reduced. This action also appears to be due to the release of noradrenaline from a store present in the ear, either in the walls of the vessels or in tissue nearby. For if a rabbit is injected with reserpine on one day, and then killed on the following day, the noradrenaline which can be extracted from the skin of the ear has disappeared, and when the ear is perfused with
Ringer's solution, nicotine no longer causes vasoconstriction. When the skin of such an ear is extracted, no noradrenaline is found present in it. Some individuals suffer from a disease called thrombo-angiitis obliterans which when active causes such a restriction of the circulation through the fingers that the tissues become dry and bloodless, and the finger tips may become gangrenous. The disease may be quiescent for long periods; but if the patient begins to smoke, the symptoms get worse and the disease becomes active. It seems likely that the effect of smoking is explained by nicotine releasing noradrenaline from the neighbourhood of the small vessels.

The exact site of storage of noradrenaline in the heart and in or near the vessels is unknown. There is a possibility that it is held in tissue known as chromaffin tissue. These are cells which stain brown with potassium dichromate; they form the greater part of the cells of the adrenal medulla. Such cells have long been known to oceur among the fibres of the sympathetic system, and side by side with the sympathetic ganglion cells. They originate in the neural crest like the sympathomimetic ganglion cells and were called by Kohn "paraganglia" in 1903. Evidence is beginning to accumulate that the distribution of chromaffin cells is much wider. Busacchi in 1912 described a "paraganglion cardiacum" lying around the origins of the coronary arteries. AdamsRay and Nordenstam (1956) have described such cells in human skin, and Leach believes that they may be present in the skin of the rabbit ear, in the skin of the cat's tail and in the nictitating membrane of the cat's eye. In all these situations nicotine exerts an action on the normal tissue. Thus, as already mentioned, nicotine causes constriction of the vessels of the rabbit ear ; it also causes erection of the hair on the cat's tail, and finally it causes contraction of the nictitating membrane when isolated from the body and free from ganglia. Yet if an animal is previously treated with reserpine, and if in consequence all local stores of noradrenaline disappear, nicotine loses these actions, and all the chromaffin cells are lost. It may therefore be that nicotine exerts its peripheral effects by discharging noradrenaline from chromaffin cells.
J. H. BuRN

\section{THE NEW ZEALAND ANTARCTIC EXPEDITION}

$I^{\mathrm{N}}$ the thirty-third Thomas Cawthron Memorial Lecture, Sir Edmund Hillary described some of the experience gained by members of the New Zealand Antarctic Expedition (Cawthron Institute, Nelson, New Zealand. Thomas Cawthron Memorial Lecture No. 33 (1958) : The New Zealand Antarctic Expedition. By Sir Edmund Hillary. Pp. $12+4$ plates. Nelson: Cawthron Institute, 1959). Although, for economy, the expedition made use of Ferguson tractors, Sir Edmund records that, with three 'Snocats', the journey could have been done "with twice the comfort and in almost half the time".

Comparison of the buildings which have been traditionally used on British expeditions and the newer module type of construction shows the advantages to rest almost entirely with the latter.

The experience gained with the dog teams should be of value to other expeditions. At the base, the dogs lived primarily on seal meat supplemented by vitamin pills, although this was varied at times with tinned dog food. In the field lightness was of primary importance and here pemmican was used. There were two forms of pemmican. One was the standard 'Bovril' pemmican which has been used a great deal in the Antarctic and the Arctic. Long and strenuous trips have been made on this pemmican, but the literature on the subject is filled with criticisms of it and descriptions of the deterioration in dog health when it is used. The Expedition's experience with 'Bovril' pemmican was much the same--loss of condition and continual scouring. "It was very fortunate that we also had generous supplies of the second type of pemmican, which was based on a formula developed by Dr. Malcolm of the Otago Medical School for the Byrd Expedition". 'This pemmican proved to be an outstanding success and 